\title{
Solid Pseudo-Papillary Neoplasm of the Pancreas (SPN): A Single Institution Annual Report
}

\author{
Dr. Arjun Athmaram, Dr. Basila Ameer Ali, Dr. Harishchandra B
}

\begin{abstract}
Solid pseudopapillary neoplasm of pancreas (SPN) is a rare entity, seen mostly in young females. We report 3 cases of SPN over 1 year in Yenepoya Medical College Hospital, Deralakatte, Mangalore .All cases presented in asymptomatic young females, underwent surgical resection with $100 \%$ survival. Almost all the cases of SPN have been reported only in the past decade. Surgery is the mainstay of treatment. All the three cases reported in under 1 year in a single institution is alarming.
\end{abstract}

Keywords: Solid Pseudopapillary neoplasm of Pancreas, SPN, Pancreatic Neoplasm

\section{Introduction}

Solid pseudopapillary neoplasm of pancreas (SPN) is a rare entity, seen mostly in young females. Features include low grade malignancy, rare metastasis, full curative resection, and excellent prognosis.

More than two thirds of all reported cases were in the last ten years.

\section{Patients and Methods}

A retrospective analysis of all patients diagnosed and treated for Solid Pseudopapillary neoplasm of the pancreas (SPN) from November 2014 to November 2015 was carried out.

The clinical, radiological, operative and survival data were obtained and analysed.

In all three patients were identified.

\section{Case 1}

A healthy 22 yo woman, being evaluated for infertility, was found to have a growth in the pancreas during routine evaluation.

Asymptomatic.

Physical examination unremarkable.

Routine investigations including LFT and pancreatic enzymes normal.

Tumour markers namely CEA, CA19-9, CA125 normal.

USG abdomen revealed a hyperechoic lesion in the tail of the pancreas.

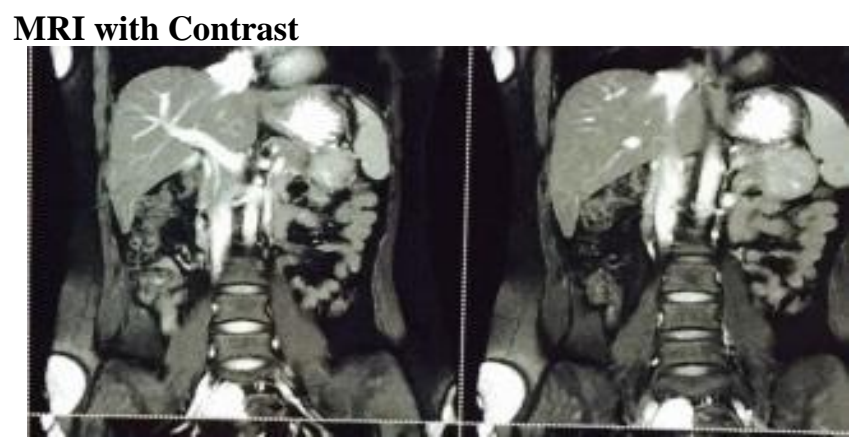

MRI revealed a growth in the tail end of the pancreas of about $5 \times 4 \mathrm{cms}$ with solid and cystic areas. No evidence of metastasis.
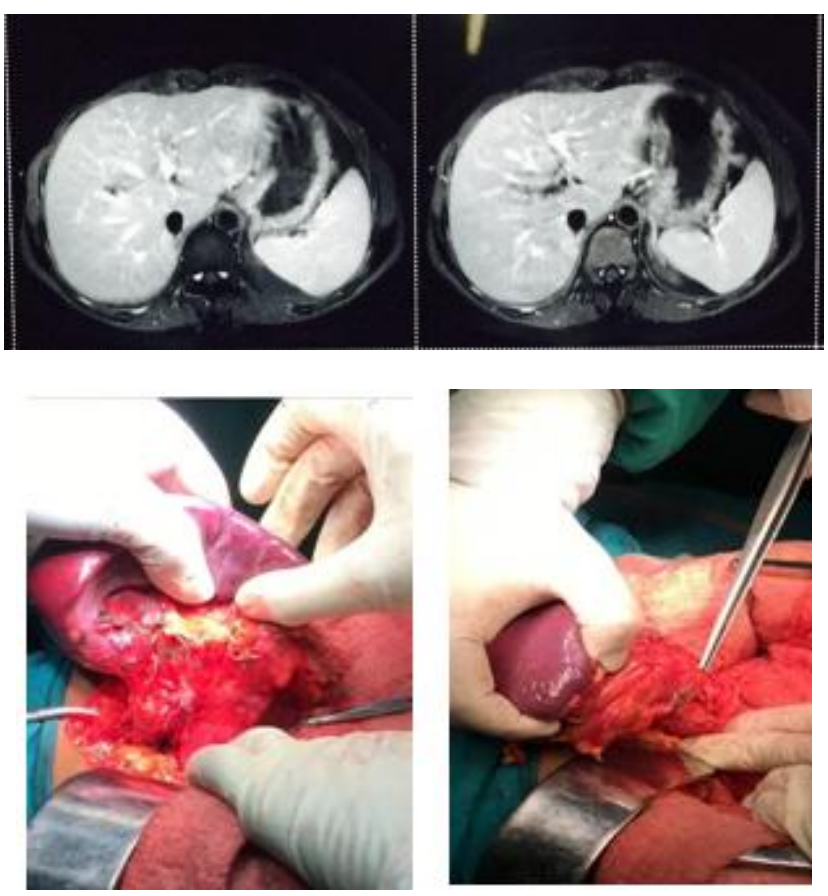

Distal pancreatectomywith splenectomy under GA.

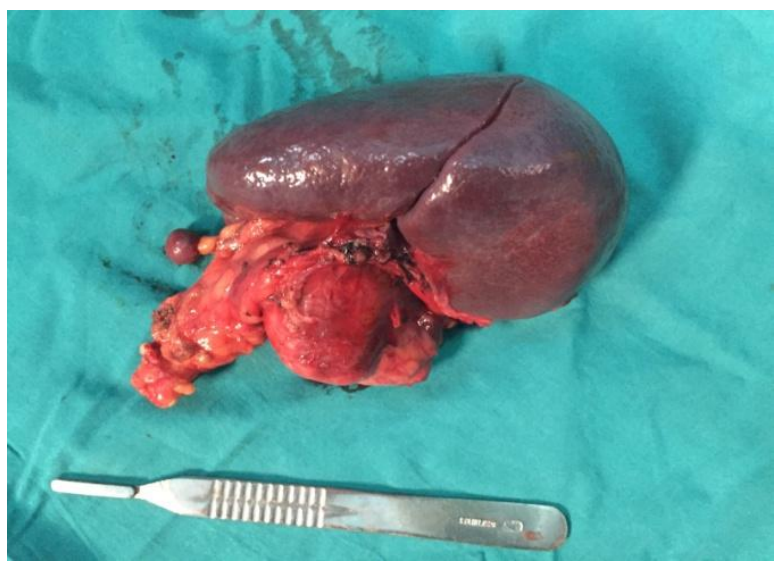

Resected tumor with spleen 


\section{International Journal of Science and Research (IJSR) \\ ISSN (Online): 2319-7064}

Index Copernicus Value (2013): 6.14 | Impact Factor (2015): 6.391

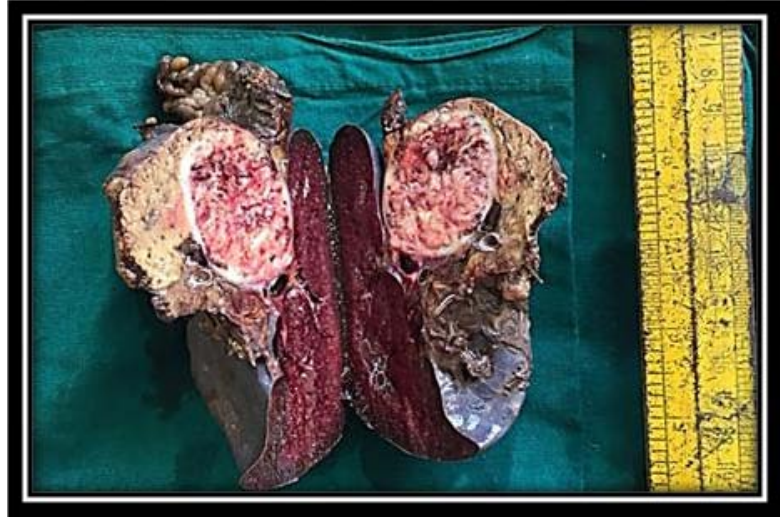

Gross Cut Section of the tumor

\section{Follow up}

It was confirmed it to be a Solid psuedopapillary neoplasm (SPN) of the pancreas histopathologically. She did not require post op chemotherapy. On regular follow up.

\section{Case 2}

25 year old woman, presented with upper abdominal pain. Physical examination unremarkable, except for tenderness. Routine blood investigations normal.

USG abdomen revealed a hyperechoic mass in the body of the pancreas.

Tumour markers were normal

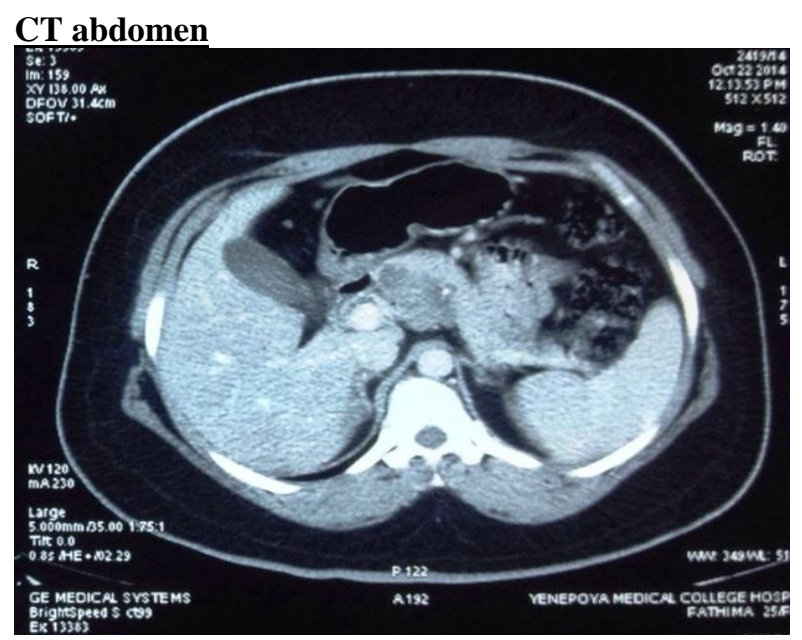

Heterogeneously enhancing well defined solid and cystic lesion in the neck and body.

\section{CT guided FNAC confirmed the diagnosis of SPN}

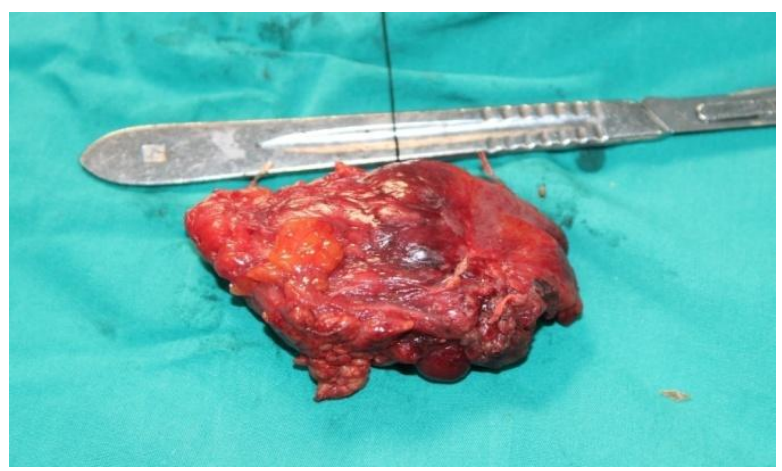

Central pancreatectomy with Roux-en-y distal pancreaticojejunostomy.

\section{Follow up}

Histopathological features suggestive of SPN with presence of perineural and vascular invasion, and local invasion into surrounding pancreatic tissue suggesting malignant behaviour.

Neuron specific enolase and Vimentin strongly positive.

She was referred to an oncologist and was started on Chemotherapy, Gemcitabine.

She is on regular follow up.

\section{Case 3}

19 year old girl, was admitted for vague upper abdominal pain and fullness.

- No other symptoms.

- Physical examination unremarkable.

- Routine blood investigations normal.

- USG abdomen revealed a hyperechoic mass in the tail of the pancreas, requiring further evaluation.

- Tumour markers WNL.

- CT abdomen revealed a hypodense cystic lesion with solid components $5 \times 4 \mathrm{cms}$ seen arising from the tail of the pancreas, suggestive of SPN

- She underwent distal pancreatectomy with splenectomy.

This was reported as Solid Pseudopapillary neoplasm of pancreas (SPN) histopathologically.

She was referred to an oncologist, who opined that she did not require adjuvant therapy.

On regular follow up.

\section{Results}

- Three cases of SPN

- Female sex

- Mean age: 22 years

- Presentation: non specific

- Site: Tail of pancreas-2, body-1

- Mean size: $5 \mathrm{cms}$

- Local invasion- 1

- Metastasis- Nil

- Surgery: Distal pancreatectomy+splenectomy-2, Central pancreatectomy roux-en-y pancreatico-jejunostomy-1

- Follow up: adjuvant therapy-1

- Mortality: 0\%

- Survival: $100 \%$ short follow up, 1 year for 1 patient, and 3 months for 2 patients.

\section{Discussion}

- Dr. V. K. Frantz first described SPN in 1959.

- Nomenclature: WHO in 1996 accepted the term Solid Psuedopapillary neoplasm of the pancreas.

- $0.13 \%-2.7 \%$ of all pancreatic tumours.

- Usually associated with a low potential for malignancy, indolent and better prognoses.

- Young women, 10:1 ratio and age $<35$ years, rare instances above 50 years

- Few cases reported in males, and usually in older males and more aggressive tumours. 


\section{International Journal of Science and Research (IJSR) \\ ISSN (Online): 2319-7064}

Index Copernicus Value (2013): 6.14 | Impact Factor (2015): 6.391

\section{Etiology}

- Definite etiology unknown

- Various attempts to correlate the immunoprofile of tumours have failed

- Origin from 'primordial' pancreatic cell lines, (pleuripotetent cells)

- Most consistently positive markers (>90\%)

- Vimentin

- NSE

- $\alpha 1$-antitrypsin

- $\alpha 1$-antichymotrypsin

- Progesterone receptors

\section{Presenting complaints}

- Clinically, vague abdominal symptoms with fullness or discomfort

- Abdominal pain, nausea, fullness are mainly due to bulky tumour (mean size $11 \mathrm{cms}$ ) compressing local structures in the upper abdomen.

- Palpable abdominal mass.

\section{Examination}

- Mostly normal

- Upper abdominal mass

- Tenderness

\section{Investigations}

- Routine blood investigations WNL, LFT, Pancreatic enzymes WNL

- Tumour markers WNL

- Radiologic imaging essential part of the diagnosis of pancreatic SPN.

- USG abdomen.

- In CT scan

- No enhancement of the cystic portions

- Slight heterogeneous enhancement of the solid portions in the arterial phase and marked enhancement in the portal venous phase.

- Intravenous contrast enhancement inside the mass suggests haemorrhagic necrosis

- MRI is better than CT for distinguishing certain tissue characteristics, such as

- Haemorrhage

- Cystic degeneration

- Presence of capsule (indicated by high signal intensity on T1-weighted imaging and slightly progressive heterogeneous peripheral contrast enhancement) seen after gadolinium.

- Angiography usually demonstrates an avascular or hypovascular pancreatic tumour

- Delineate the mass from other involved and adjacent structures.

- Guided FNAC : confirmatory test for diagnosing SPN, done if necessary.

\section{Management}

Surgical resection mainstay of treatment, options are limited surgery: distal pancreatectomy (with or without splenectomy), central pancreatectomy, enucleation.

Major resection if extensive local spread: total pancreatectomy, pancreaticoduodenectomy,liver resection, metastatectomy.

Significant as prognosis is excellent after curative resection.

Size of the lesion not a criteria for resectability.

Metastasis to liver - not a contraindication to resection.

Tumours with synchronous metastasis to liver have been resected and have shown good response.

\section{Histopathology}

Grossly, well encapsulated, 8-10 cms

Cut surface shows large spongy areas of haemorrhage alternating with solid and cystic degeneration

Microscopically, pseudopapillae are microvascular stalks, covered by several layers of epithelial cells which are discohesive and appear to be falling off at the periphery.

\section{HPE}
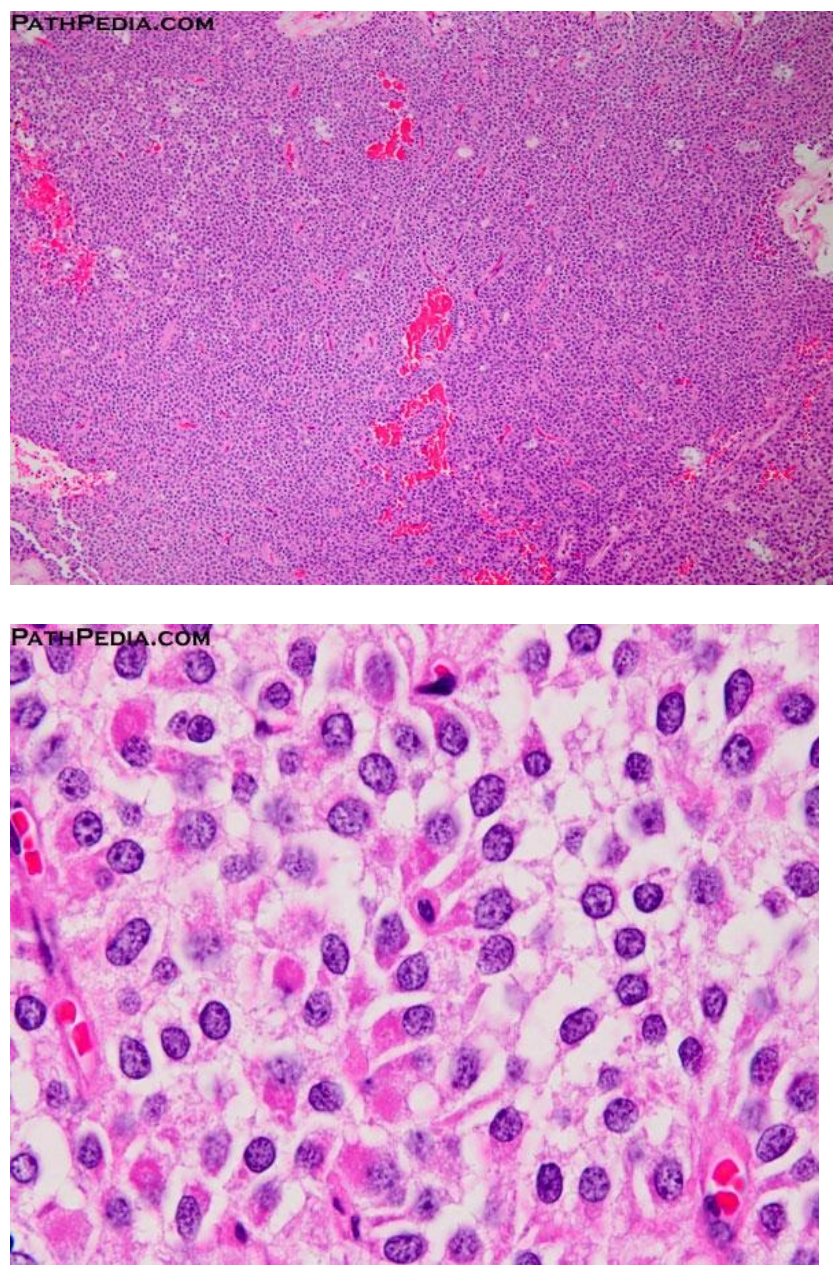


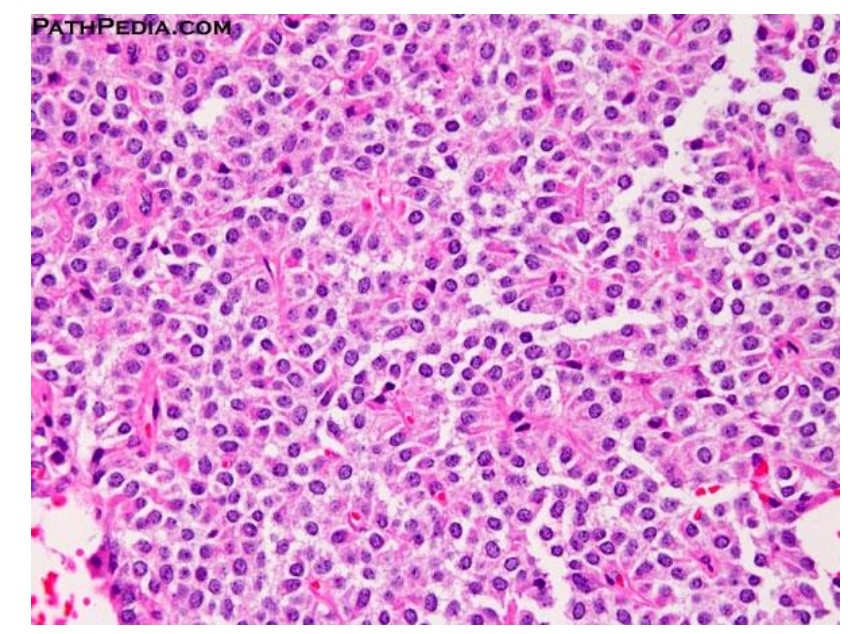

\section{Malignant potential}

- Angioinvasion

- Perineural invasion

- Deep invasion of the surrounding pancreatic parenchyma.

- Venous invasion

- Degree of nuclear atypia

- Mitotic count

- Prominence of necrobiotic cell nests

\section{Prognosis}

- Classical SPN has a good prognosis with full curative resection.

- 5 year survival is $98.8 \%$.

- 5 year disease free survival is $95.4 \%$.

- Metastasis usually occurs to liver (15\%) and/or peritoneum, usually synchronous, not metachronous.

- Metastasis may be a feature of advanced age of the patient or of the tumour cells itself.

\section{Predictive factors for recurrence}

- Tumour size $>8 \mathrm{cms}$

- Microscopic features of malignancy

- Metastatic disease

- Neoadjuvent therapy is by chemotherapy with gemcitabine, adriamycine.

\section{Significance}

THREE cases of SPN diagnosed at this hospital in $<1$ year.

\section{References}

[1] Frantz VK. Tumours of the pancreas. In: Bumberg CW (ed). Atlas of tumour pathology, VII. Fascicles 27 and 28. Washington DC: Armed Forces Institute of Pathology, 1959;32-

[2] Vollmer CM, Dixon E, Grant DR. Management of pseudopapillary tumour of the pancreas with liver metastases. HPB 2003; 4: 264-7.

[3] Vincent PJ, Nagpal BM, Singh P, Harjai MM, Nagi GS, Satyanarayana S. Gruber-Frantz tumour. MJAFI 2003; 59: 355-7

[4] Patil TB, Shrikhande SV, Khanhere HA, Saoji RR, Ramadwar MR, Shukla PJ. Solid pseudopapillary neoplasm of the pancreas: a single institution experience of 14 cases. HPB 2006; 8: 148-150.
[5] Huang HL, Shih SH, Chang WH, Wang TE, Chen MJ, Chan YJ. Solid-pseudopapillary tumour of the pancreas: Clinical experience and literature review. World $J$ Gastroenterol 2005; 11; 1403-9. 